\title{
Indexing Tree and Subtree by Using a Structure Network
}

\author{
Mingming Zhang and Shinichiro Omachi \\ Graduate School of Engineering, Tohoku University \\ Aoba 6-6-05, Aramaki, Aoba-ku, Sendai-shi, 980-8579 Japan \\ johnson@aso.ecei.tohoku.ac.jp, machi@ecei.tohoku.ac.jp
}

\begin{abstract}
In pattern recognition, graphs become alluring more and more as structural pattern representations due to their richer representability than feature vectors. However, there are many challenging problems using graphs for pattern recognition. One is that it is difficult to investigate the relationships of graphs effectively, even of trees. In this paper, we focus on the structure relationship analysis of trees, such as tree and subtree isomorphism, maximum common subtree, minimum common supertree, etc., which is almost suffered from all kinds of tree recognition problems. For investigating the relationships of structures of trees, we propose a structure network to represent the evolutional relationships of structures of trees. Moreover, for a lot of tree isomorphism problems appearing in the application of structure network, we propose a method that encodes the structure of tree as a numerical sequence, and illustrate its efficiency by comparing it with traditional matching method for tree isomorphism problem.
\end{abstract}

Keywords: tree isomorphism, subtree isomorphism, tree indexing, structure analysis, tree measure.

\section{Introduction}

In pattern recognition and computer vision, the graph representation of objects has become alluring more and more along with the increase of the needs to consider the context of features. In a graph, not only the traditional features can be represented as vertices, but also the relationships defined on them can be represented as edges. Especially the tree as the simplest graph is used widely in many studies, such as data mining, computational biology, image analysis, document analysis, even in automatic theorem proving and compiler optimization [1.

However, when we are enjoying the advantages brought from the graph representation, we are suffering from some difficult problems at the same time. One is the high computational complexity; there exist many NP-complete or NP-hard problems in graph and tree problems [2]. Moreover, many efficient traditional methods working on the feature vectors can not be used on the graph and tree patterns straightforwardly [3. Since matching graphs or trees directly is impractical when considering their computational complexity, many approximation methods are discussed, such as tree edit distance 114, spectrum-based 
methods [5]6], common supergraph methods [7], and kernel methods [8]. In all of these methods, the tree edit distance methods are studied very widely in practices 910, and are extended to kernel methods 11, pair HMM methods 12, etc. On the other hand, in order to convert the tree classification problems to traditional classification problems, some approaches that embed trees into vector space, based on the dissimilarity computed by edit distance [13/14] or based on the spectrum of tree [15] had been proposed. Furthermore, by selecting a number of candidates from database with indexing methods [16 17] before matching them one by one, the times of matching can be reduced greatly.

In all of these problems of trees, a basic problem is the relationship analysis of tree structures, such as isomorphism, subtree or supertree, minimum common supertree, maximum common subtree, etc. Even more, in edit distance algorithm, as a sub-problem in the dynamic program, it is necessary to investigate all the matching possibility of common subtrees. Although tree isomorphism can be determined by matching method, no method can measure the structure of tree by numerical value clearly. In this paper we focus on these tree problems, and try to address the relationships analysis of tree structures by defining a structure map. Furthermore we also propose a method for encoding the structure of a tree.

The rest of this paper is structured as follows. In section 2 we discuss how to construct the structure network for addressing the relationship analysis of tree structures. In section [3, by clustering the vertices in a tree, we give a method to encode the structure of tree that can be used to query a tree by its structure in database or in the structure network. In section 4 we state experiments for identifying the effectiveness of the proposed encoding method. Finally we will discuss some potential applications, and give our conclusion in section 5 .

\section{Structure Network}

In this section, we discuss how to construct the structure map. First, we give some definitions and algorithms that are used throughout this paper. The trees considering in this paper are unlabeled and undirected trees. The graph isomorphism is defined as follow.

Definition 1. Two graphs $G_{1}=\left(V_{1}, E_{1}\right), G_{2}=\left(V_{2}, E_{2}\right)$ are isomorphic if and only if there is a bijection $\varphi$ between $V_{1}$ and $V_{2}$ such that for every pair of vertices $i, j \in V_{1},(i, j) \in E_{1}$ if, and only if, $(\varphi(i), \varphi(j)) \in E_{2}$.

In this paper, when two trees are isomorphic we will say that their structures are equal or they have the same structure. To determine whether two unlabeled trees $T_{1}$ and $T_{2}$ are isomorphic, we use the algorithm introduced by Kucera in [18] as shown bellow:

1. In each tree, label all the leaves with 1 . If the numbers of leaves in $T_{1}$ and $T_{2}$ do not coincide, stop with "non-isomorphic".

2. In each tree, determine the sets of unlabeled vertices $S_{1}$ and $S_{2}$ such that every neighbor of $v \in S_{i}$, except at most one, has a label. Tentatively, label 


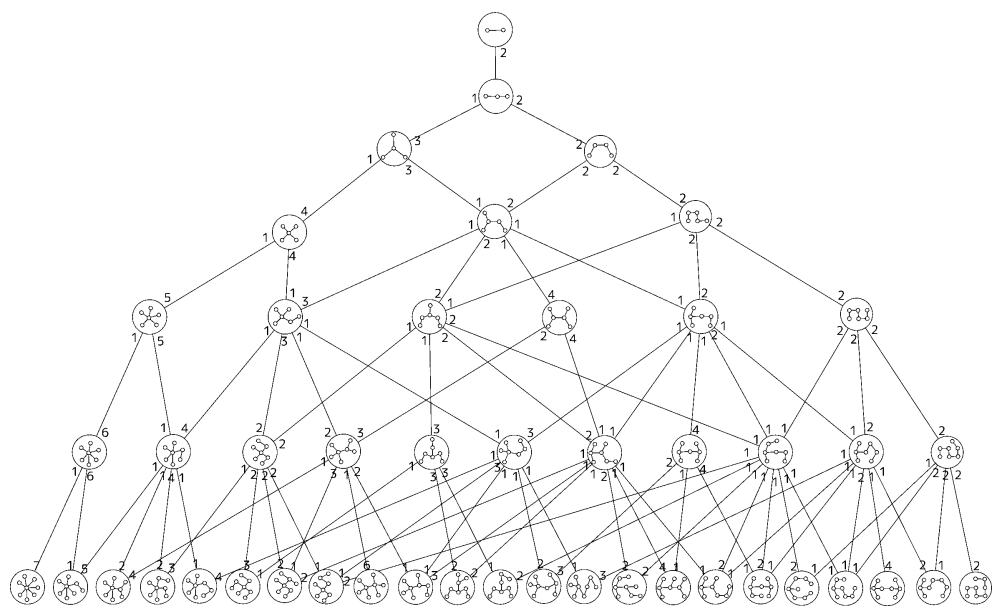

Fig. 1. Structure map under 8 vertices

$v$ with the list $\left(l_{1}, \cdots, l_{k}\right)$ of labels of its labeled neighbors, sorted in nondecreasing order. Compare the respective tentative labels for the vertices in $S_{1}$ and $S_{2}$. If these labels do not agree (as multi-sets), stop with "nonisomorphic".

3. Substitute the tentative labels (which are lists of numbers) by new labels which are just numbers $k, k+1, k+2, \cdots$ in an arbitrary way. The only restriction is that vertices with the same tentative labels should get the same new labels, and that the labels $k, k+1, k+2, \cdots$ have not been used before.

4. If not all vertices have labels, go back to step 2 .

5. Stop with "isomorphic".

As stated in section 1, usually, except for determining the isomorphism problem between two trees, we also need to deal with other relationships between trees. Although for subtree isomorphism problem Shamir gives an efficient matching method in [19], matching every pair of trees in a database is a very hard work when only using matching methods. So in this section we propose a method to construct a structure network, and discuss how to joint a structure of tree into this network. An example of this structure network under 8 vertices is shown in Fig. 1. In the structure network, each structure is drawn as a node that can indicate a cluster of trees with the same structure. All nodes of the network are organized into layers by their size. The edge indicates a sub-super relationship and only the adjacent layers can be linked. The numbers around each node will be discussed later.

There are two ways to construct the structure network, one is a top-down way that evolve the network from a two vertices structure. Another is a bottomup way. When a tree is given, if its structure is outside of this network, then decompose it bottom-up until it can be completely connected into the network. 


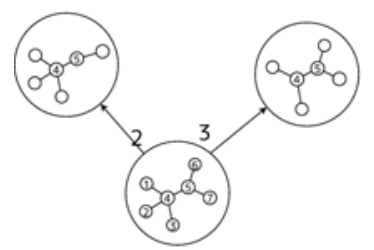

(a)

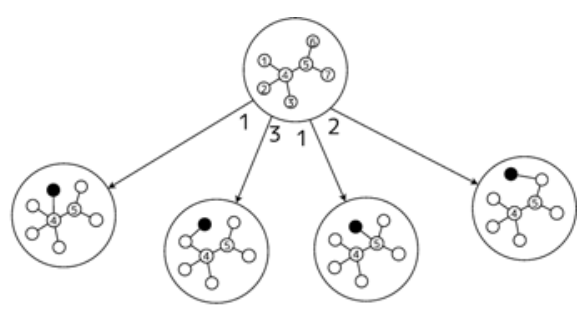

(b)

Fig. 2. The probability of reduction(a) and growth(b) of a topolgy

For a node in the network, there are two related processes; one is reduction: by removing a leaf in it, it can reduce to a sub-structure, and the other one is growth: by inserting an edge into a structure, it can grow up to a super-structure. First, let us consider the number that how many sub-structures can reduce to from a structure and the number that how many super-structures can grow up to. In general, the number of sub-structure that can reduce to is equal to the number of categories of its leaves. Inversely, the number of super-structures that can grow up to is equal to the number of categories of all vertices. For example, considering the structure shown in Fig. 2. (a) shows the reduction, and (b) shows the growth. In this case all of the vertices can be clustered into 4 categories, and two of them are leaves.

$$
\{1,2,3\},\{4\},\{5\},\{6,7\}
$$

For the clusters of leaves, their sizes are 3 and 2, which indicate the probabilities reducing to these two kinds of sub-structure when removing a leaf randomly. Similarly, the size of each cluster indicates the probability growing to each supertopology when inserting an edge randomly. Here we can cluster vertices by using their histogram of graph distance. Firstly for a vertex compute the graph distances from it to all the other vertices, and use a histogram diagram to count the distribution of distances. Such histograms of vertices in Fig. 2 are shown in Fig. 3. The histograms of vertices $1,2,3$ are (a), 4 is (b), 5 is (c), 6,7 are (d). Then by using these histograms we can cluster vertices and find out the super-structures and sub-structures in up and down layers efficiently.

The top-down algorithm to construct the structure network until size $N$ is shown as bellow. $T^{(N)}$ denotes a structure with $N$ vertices.

initialize the structure network $S=\phi$;

initialize the two-vertices structure $t^{(2)}$ and add it into $S$;

for each $i$ from 2 to $N-1$; do

for each structure $t_{\text {sub }} \in T^{(i)}$, where $T^{(i)} \subseteq S$; do

cluster all vertices in $t_{s u b}$;

for each cluster $C$ do

select an arbitrary vertex $v \in C$; 


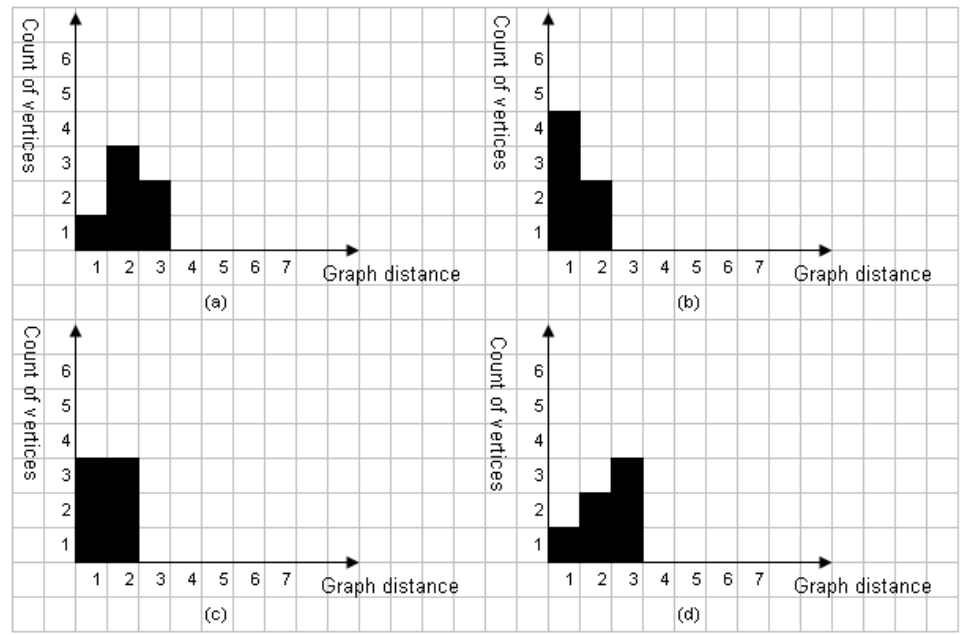

Fig. 3. Four kinds of histogram of vertex graph distance

add an edge connecting to $v$ to construct a new structure $t_{n e w}$; look for the isomorphic structure $t_{\text {sup }}$ of $t_{\text {new }}$, where $t_{\text {sup }} \in T^{(i+1)}$ and $T^{(i+1)} \subseteq S$

if found then else

link the structure $t_{\text {sub }}$ to $t_{\text {sup }}$;

add $t_{n e w}$ into $S$;

link the structure $t_{\text {sub }}$ to $t_{n e w}$; end if done;

done;

done;

When a given structure indicated by $t^{(N)}$ is not in the network $S$, a bottom-up algorithm used to joint it into the structure network is:

look for the isomorphic structure $t_{\text {sup }}$ of $t^{(N)}$, where $t_{\text {sup }} \in T^{(N)}$ and $T^{(N)} \subseteq S$; if found then

else

$$
\text { return } t_{\text {sup }}
$$

add a new cluster into $S$ and use $t^{(N)}$ to indicate it;

for each $t_{\text {sup }} \in S$ where $t_{\text {sup }}$ have no link to the structure in $T^{\left(\text {Size }\left(t_{\text {sup }}\right)-1\right)}$; do

cluster all leaves in $t_{\text {sup }}$;

for each cluster $C$; do

select an arbitrary leaves $v \in C$;

remove $v$ to construct a new structure $t_{n e w}$; 
look for the isomorphic structure $t_{\text {sub }}$ of $t_{n e w}$, where $t_{\text {sub }} \in T^{\left(\text {size }\left(t_{n e w}\right)\right)}$ and $T^{\left.\left(\text {size(t } t_{\text {new }}\right)\right)} \subseteq S$;

if found then

link the structure $t_{\text {sup }}$ to $t_{\text {sub }}$; else

add $t_{\text {new }}$ into $S$;

link the structure $t_{\text {sup }}$ to $t_{n e w}$; end if done;

done;

end if;

\section{Encoding a Structure}

In this section, we focus on how to describe a structure in a numerical way. As described in section 2, generally, to determine the isomorphism of two trees the matching method given by Kucera in 18, is used. Although its time complexity is $O(n)$, since it is a matching method, in some applications such as querying a structure from a database, a large amount of matching is neccesary. For dealing with the structures of trees more efficiently, in this section we discuss how to encode the structure informations of trees. We noticed that the essence of the isomorphism algorithm in fact is a procedure of clustering vertices step by step. In another way, as shown in section 2, using the histogram of graph distances the vertices can be clustered well too. For example, let us consider two isomorphic trees shown in Fig. 4. In the isomorphism algorithm a label is computed and assigned to each node, and their correspondences are shown in Table 1 In the matching method, to decide these correspondent relationship the adjacent labels should also be considered.

In contrast, in a column in Table 1, we show their histograms of graph distances. In this way, the correspondent relationships between vertices are decided uniquely by their histograms. Furthermore, the difference from matching method is that we can use histograms to encode the topology of a tree. Due to the connectivity of the tree, the histogram is also connected. That is for an arbitrary

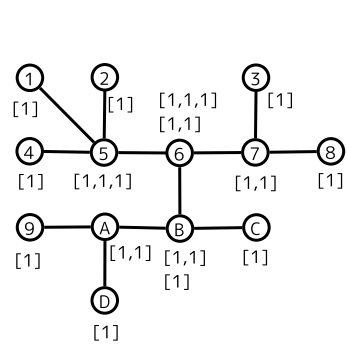

(a)

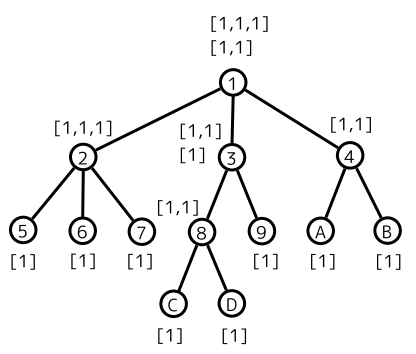

(b)

Fig. 4. Example of the isomorphic matching algorithm for Tree (a) and (b) 
Table 1. The correspondence between vertices and their histograms

\begin{tabular}{|l|l|l|l|}
\hline Vertices in Tree (a) & Vertices in Tree (b) & Label & Histogram \\
\hline $1,2,4$ & $5,6,7$ & {$[1]$} & {$[1,3,2,4,2]$} \\
\hline $9, \mathrm{D}$ & $\mathrm{C}, \mathrm{D}$ & {$[1]$} & {$[1,2,2,2,5]$} \\
\hline 3,8 & $\mathrm{~A}, \mathrm{~B}$ & {$[1]$} & {$[1,2,2,5,2]$} \\
\hline $\mathrm{C}$ & 9 & {$[1]$} & {$[1,2,4,5]$} \\
\hline 5 & 2 & {$[1,1,1]$} & {$[4,2,4,2]$} \\
\hline $\mathrm{A}$ & 8 & {$[1,1]$} & {$[3,2,2,5]$} \\
\hline 7 & 4 & {$[1,1]$} & {$[3,2,5,2]$} \\
\hline $\mathrm{B}$ & 3 & {$[[1,1],[1]]$} & {$[3,4,5]$} \\
\hline 6 & 1 & {$[[1,1,1],[1,1]]$} & {$[3,7,2]$} \\
\hline
\end{tabular}

distance $i$, if $i$ th element of the histogram is not zero, then the $(i-1)$ th element is not zero too. So we can use a sequence to denote the histogram only including the nonzero element namely histogram sequence. Using this histogram sequence we can encode each cluster to a field defined as:

$$
\begin{aligned}
h & :=\text { histogram sequence; } \\
l & :=\text { lengh of } h \\
s & :=\text { size of cluster; } \\
\text { field } & :=(l+2), s, h
\end{aligned}
$$

Finally, sort all cluster fields alphabetically, and link them to compose a long sequence. This long sequence is the final result of encoding the structure of a tree, and it includes all the clustering informations of vertices obviously. For example, the structure of the tree in Fig. 4 can be encoded as

$$
\begin{gathered}
(\mathbf{5}, 1,3,4,5, \mathbf{5}, 1,3,7,2, \mathbf{6}, 1,1,2,4,5, \mathbf{6}, 1,3,2,2,5, \mathbf{6}, 1,3,2,5,2, \\
\mathbf{6}, 1,4,2,4,2, \mathbf{7}, 2,1,2,2,2,5, \mathbf{7}, 2,1,2,2,5,2, \mathbf{7}, 3,1,3,2,4,2) .
\end{gathered}
$$

In the algorithm proposed in section 2, by replacing the matching method to the encoding method where the step is looking for isomorphic structure, the algorithm could be more efficient.

\section{Experiments}

In this section, we will verify the effectiveness of the encoding method and discuss the indexing tree and subtree by structure network. We first create a database that contains about 20,000 unlabeled and undirected trees with up to 20 vertices.

\subsection{Experimental Settings}

In this section, we address the algorithm used to create the database of trees. Here we use $T^{(j)}$ to denote the set of $j$ vertices trees, and $T_{i}^{(j)}$ denotes its element. The algorithm is: 
prepare $T^{(2)}$;

foreach $j$ from 2 to 20 do

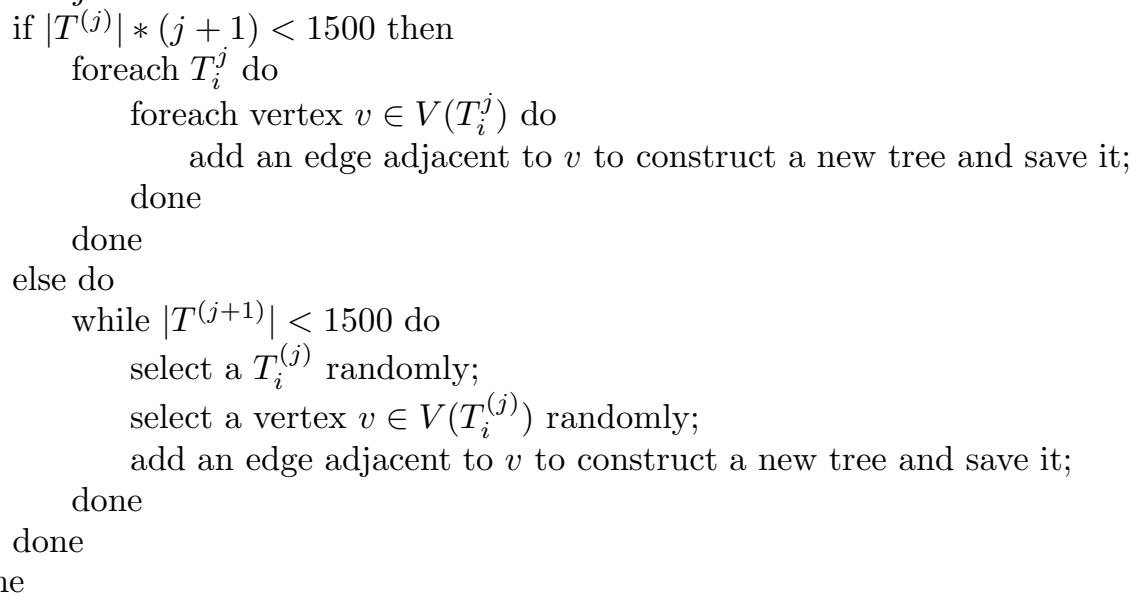

With this algorithm, we made a tree database including 19,410 trees. These trees are clustered into 8,251 categories by using the isomorphism algorithm. In these clusters, 2,898 clusters which include more than one tree.

\subsection{Results}

Firstly, we cluster all the trees in database by using the proposed encoding method. Then the result is compared with the one of the isomorphism algorithm. As shown in Table.2, the clustering result of the proposed encoding method is the same as the isomorphism algorithm. In concern with the computing complexity, in an LAM/MPI environment with 10 nodes(CPUs), clustering this database by using isomorphism algorithm spent about 9.5 days. In contract, computing all the encoding sequence only needs 45 minutes.

For the structure network, because constructing it is based on the encoding method or isomorphism algorithm, the correctness of clustering is clear. With it, we discuss the time and spatial complexity of construction. In Fig. 5 we show three kinds of rough statistic data of constructing the structure network of under 15 vertices by using top-down algorithm. In Fig. 5 (a) we show the raise of the number of nodes and edges along with the size of tree. In Fig. 5] (b) the computing time is shown. Although it is expensive to construct the structure network, once the network is be constructed offline, to investigate the relationships between trees can be achieved by only using shortest path algorithm. Moreover, by using the bottom-up algorithm, it is possible to construct the structure network partially.

Table 2. The results of experiments

\begin{tabular}{|l|l|}
\hline proposed method & Encoding Index \\
\hline correct rate & $100 \%$ \\
\hline
\end{tabular}




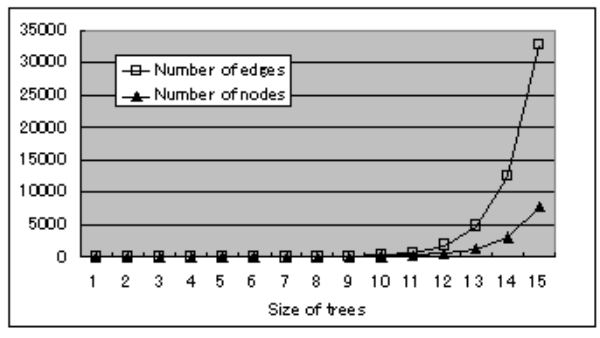

(a)

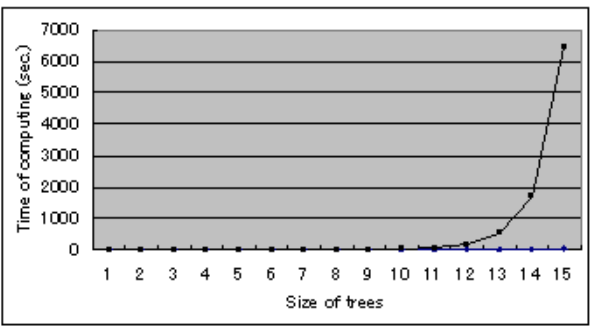

(b)

Fig. 5. Complexity of constructing the structure network:(a) the number of nodes and edges (b) the computing times

\section{Conclusion}

In this paper, we proposed a method to construct a structure network for indexing tree and subtree, and based on the clustering vertices of a tree, we proposed an encoding method to indicate the structure of a tree as a numerical sequence for indexing. As shown in the results of experiment, we can conclude the effectiveness of the proposed methods. Furthermore, tree edit distance algorithm is exploited widely in the applications as stated in section 1, Since each edit operator in tree edit distance algorithm is related to a link in the structure network, by defining proper cost functions on the edges and nodes of structure network it is possible to find out the optimal edit path efficiently on the structure network for two trees. On the other hand, since each edge of structure network is related to two probabilities according to two different directions, it is possible to compute a conditional probability between two structure and the joint probability of them by proability propagation. For future work, first we are going to investigate the encoding method in detail for a more compact index. In application side, we will try to combine the structure network with the tree edit distance to improve the efficiency of tree edit distance algorithm or to develop the structure network to a Bayesian network for recognition of tree patterns.

\section{References}

1. Bille, P.: A survey on tree edit distance and related problems. Theoretical Computer Science 337(1-3), 217-239 (2005)

2. Köbler, J., Schöning, U., Torán, J.: The graph isomorphism problem: its structural complexity. Springer, Heidelberg (1993)

3. Bunke, H., Irniger, C., Neuhaus, M.: Graph matching - challenges and potential solutions. In: Roli, F., Vitulano, S. (eds.) ICIAP 2005. LNCS, vol. 3617, pp. 1-10. Springer, Heidelberg (2005)

4. Klein, P.N., Sebastian, T.B., Kimia, B.B.: Shape matching using edit-distance: an implementation. In: SODA, pp. 781-790 (2001) 
5. Wilson, R.C., Zhu, P.: A study of graph spectra for comparing graphs and trees. Pattern Recognition 41(9), 2833-2841 (2008)

6. Wilson, R., Hancock, E., Luo, B.: Pattern vectors from algebraic graph theory. IEEE Ttansactions on Pattern Analysis and Machine Intelligence 27(7), 1112-1124 (2005)

7. Bunke, H., Foggia, P., Guidobaldi, C., Vento, M.: Graph clustering using the weighted minimum common supergraph. In: Hancock, E.R., Vento, M. (eds.) GbRPR 2003. LNCS, vol. 2726, pp. 235-246. Springer, Heidelberg (2003)

8. Kashima, H., Tsuda, K., Inokuchi, A.: Marginalized kernels between labeled graphs. In: Fawcett, T., Mishra, N. (eds.) ICML, pp. 321-328. AAAI Press, Menlo Park (2003)

9. Sebastian, T.B., Klein, P.N., Kimia, B.B.: Recognition of shapes by editing their shock graphs. IEEE Trans. Pattern Anal. Mach. Intell. 26(5), 550-571 (2004)

10. Neuhaus, M., Bunke, H.: Self-organizing maps for learning the edit costs in graph matching. IEEE Transactions on Systems, Man, and Cybernetics, Part B 35(3), 503-514 (2005)

11. Neuhaus, M., Bunke, H.: Edit distance-based kernel functions for structural pattern classification. Pattern Recognition 39(10), 1852-1863 (2006)

12. Sakakibara, Y.: Pair hidden markov models on tree structures. In: ISMB (Supplement of Bioinformatics), pp. 232-240 (2003)

13. Torsello, A., Hancock, E.R.: Graph embedding using tree edit-union. Pattern Recognition 40(5), 1393-1405 (2007)

14. Riesen, K., Neuhaus, M., Bunke, H.: Graph embedding in vector spaces by means of prototype selection. In: Escolano, F., Vento, M. (eds.) GbRPR. LNCS, vol. 4538, pp. 383-393. Springer, Heidelberg (2007)

15. Bai, X., Hancock, E.R.: Heat kernels, manifolds and graph embedding. In: [20], pp. $198-206$

16. Shokoufandeh, A., Dickinson, S.J., Siddiqi, K., Zucker, S.W.: Indexing using a spectral encoding of topological structure. In: CVPR, pp. 2491-2497. IEEE Computer Society, Los Alamitos (1999)

17. Irniger, C., Bunke, H.: Decision tree structures for graph database filtering. In: $[20]$, pp. $66-75$

18. Kučera, L.: Combinatorial algorithms. Taylor \& Francis, Abington (1990)

19. Shamir, R., Tsur, D.: Faster subtree isomorphism. J. Algorithms 33(2), 267-280 (1999)

20. Fred, A., Caelli, T.M., Duin, R.P.W., Campilho, A.C., de Ridder, D. (eds.): SSPR\&SPR 2004. LNCS, vol. 3138. Springer, Heidelberg (2004) 